\title{
Modified variable phase method for the solution of coupled radial Schrödinger equations
}

\author{
S. N. Ershov* \\ Joint Institute for Nuclear Research, RU-141980 Dubna, Russia \\ J. S. Vaagen \\ Institute of Physics and Technology, University of Bergen, N-5007 Bergen, Norway \\ M. V. Zhukov \\ Fundamental Physics, Chalmers University of Technology, S-41296 Göteborg, Sweden \\ (Received 7 October 2011; revised manuscript received 23 November 2011; published 9 December 2011)
}

\begin{abstract}
A modified variable phase method for the numerical solution of coupled radial Schrödinger equations, which maintains linear independence for different sets of solution vectors, is suggested. The modification involves rearrangement of coupled equations to avoid the usual numerical instabilities associated with components of the wave function in their classically forbidden regions. The modified method is applied to nuclear structure calculations of halo nuclei within the hyperspherical harmonics approach.
\end{abstract}

DOI: 10.1103/PhysRevC.84.064308

PACS number(s): 02.30.Hq, 21.60.Gx, 24.10.Eq

\section{INTRODUCTION}

The Schrödinger equation of quantum mechanical systems is often converted into systems of coupled radial equations, with applications in nuclear physics, quantum chemistry, etc. A variety of solution methods has been developed. A widespread approach consists of two steps: First, sets of linear independent solutions are calculated and then, exploiting the linearity of the coupled equations, a suitable combination of different sets with the required boundary conditions is found. A major problem in the numerical solution of the coupled equations is the difficulty of maintaining the linear independence of the solution vectors. Problems stem from the existence of radial regions where some components of the wave function are classically forbidden and others are not. The components with negative radial kinetic energy will in general have an exponentially growing and an exponentially decreasing part. If the integration is continued through a classically forbidden region, the exponentially growing components of the wave function increase faster in the most strongly closed channels and soon start to dominate the entire wave function matrix. The small components become insignificant on the scale of the relative accuracy of the calculation. Eventually, different solutions become linearly dependent and, thus, useless for finding linear combinations with required boundary conditions. In the classically allowed region, an uneven growth of the components does not occur, since the components are mainly oscillating. But all problems involve an integration through at least one classically forbidden region, and an instability from the growth of nearly dependent solutions causes a serious numerical inaccuracy. This difficulty arises from the natural properties of solutions rather than from any particular method for their construction.

\footnotetext{
*ershov@theor.jinr.ru
}

Different methods have been suggested to maintain linear independence, such as a variety of stabilizing transformations during propagation of a solution [1-5]. Usually these transformations are rather awkward and tedious. Another approach to overcome the difficulty is to use a so-called invariant imbedding method, in which the propagated quantity is not a wave function matrix $\boldsymbol{\Psi}=\left\{\psi_{\text {in }}(r)\right\}$, but rather its logarithmic derivative $\boldsymbol{\Psi}^{\prime} \boldsymbol{\Psi}^{-1}[6-8]$ or its inverse matrix $\boldsymbol{R}=\boldsymbol{\Psi} \boldsymbol{\Psi}^{\prime-1}$ [911]. These methods have found broad application, especially for large coupled-channel calculations. In other approaches, radial wave functions are expanded in terms of orthonormal basis functions, chosen to account for some dynamical features in the most effective way. Then, a solution of differential equations is converted to a set of linear equations for expansion coefficients. Such an approach was, for example, realized in program [12].

The development of methods for the numerical integration of the Schrödinger equation, aiming at both accuracy and computational efficiency, is still an active subject of investigation (see, for example, [13,14], and references therein). Recently, we developed [15] a novel method for the solution of coupled radial Schrödinger equations, consisting of two steps. First, the full radial interval is split into finite intervals, and then the radial equations are rearranged on each interval in a way that is tailored to avoid the numerical instabilities associated with components of a wave function in their classically forbidden regions. Finally, global solutions are constructed from local ones. In the present work, the same idea of the rearrangement of coupled equations is applied to the variable phase method for solutions of Schrödinger equations. The modified method is, however, simpler and numerically more effective than that previously developed in [15]. Applications of the modified variable phase method to nuclear structure calculations within the hyperspherical harmonics approach are given for loosely bound halo nuclei. 


\section{THEORY}

The system of $N$ coupled radial Schrödinger equations may be written as

$$
\left[\frac{d^{2}}{d r^{2}}+\frac{2 m E}{\hbar^{2}}-\frac{\mathcal{L}_{i}\left(\mathcal{L}_{i}+1\right)}{r^{2}}\right] \psi_{\text {in }}(r)=\sum_{j=1}^{N} V_{i j}(r) \psi_{j n}(r),
$$

where $E$ is a total energy and $\mathcal{L}_{i}$ is the orbital angular momentum in channel $i$. The first index of $\psi_{\text {in }}(r)$ denotes the $i$ th component of a wave function $(i=1, \ldots, N)$, while the second index $n$ marks different linear independent solutions. The $N \times N$ matrix of coupling potentials $V_{i j}(r)$ is assumed to be symmetric, i.e., $V_{i j}(r)=V_{j i}(r)$. Note that the potentials include the factor $2 m / \hbar^{2}$ and have the dimension $\mathrm{fm}^{-2}$. In general, the system (1) of $N$ linear differential equations of the second order has $2 N$ linearly independent solutions called fundamental ones; $N$ solutions have a regular behavior at the origin, while $N$ others have an irregular behavior. Any solution of the system (1) can be written as a linear combination of these fundamental solutions. Only solutions that satisfy definite boundary conditions imposed at the origin and at the infinity have physical meaning. At the origin, the boundary condition demands that wave functions have the regular behavior

$$
\psi_{\text {in }}(r \rightarrow 0) \rightarrow 0
$$

while at the infinity [assume $V_{i j}(r \rightarrow \infty) \rightarrow 0$ ] the boundary condition depends on the sign of energy $E$. For bound states $(E<0)$, the problem is of the eigenvalue type and for any given eigenvalue $\left(E_{n}\right)$, solutions of (1) decay exponentially for large values of $r$,

$$
\psi_{\text {in }}(r \rightarrow \infty) \rightarrow \exp \left(-k_{n} r\right)
$$

where $k_{n}=\sqrt{2 m\left|E_{n}\right| / \hbar^{2}}$. For continuum states $(E>0)$, solutions oscillate at infinity,

$$
\psi_{\text {in }}(r \rightarrow \infty) \rightarrow \frac{l}{2}\left[H_{\mathcal{L}_{i}}^{(-)}(k r) \delta_{i n}-H_{\mathcal{L}_{i}}^{(+)}(k r) S_{i n}\right],
$$

where $k=\sqrt{2 m|E| / \hbar^{2}}$. Here, $H_{\mathcal{L}_{i}}^{( \pm)}(x)=G_{\mathcal{L}_{i}}(x) \pm{ }_{\imath} F_{\mathcal{L}_{i}}(x)$ are the Coulomb functions of the index $\mathcal{L}_{i}$ [16] describing inand outgoing spherical waves. $F_{\mathcal{L}_{i}}(x)$ and $G_{\mathcal{L}_{i}}(x)$ are regular and irregular Coulomb functions, respectively, with $l=\sqrt{-1}$. $S_{i n}$ is the $S$-matrix element for the outgoing amplitude in channel $i$ from an incoming wave in channel $n$.

The general method to solve the boundary value problem for coupled equations (1) is to construct a set of linear independent solutions and then find a linear combination of these solutions which satisfies the required asymptotic behavior. To find a set of solutions, we will apply the variable phase method that requires the knowledge of the solutions for some simplified system. To this end, two linear independent solutions $f_{i}(x)$ and $g_{i}(x)$ of the free Schrödinger equation are selected,

$$
\left[\frac{d^{2}}{d x^{2}} \pm 1-\frac{\mathcal{L}_{i}\left(\mathcal{L}_{i}+1\right)}{x^{2}}\right]\left[\begin{array}{l}
f_{i}(x) \\
g_{i}(x)
\end{array}\right]=0,
$$

where $x=k r$. The different signs before 1 in (5) correspond to positive or negative energy $E$. Free solutions are normalized by demanding that the Wronskian $W\left(f_{i}, g_{i}\right)=$ $f_{i}(x) g_{i}^{\prime}(x)-f_{i}^{\prime}(x) g_{i}(x)=-1$. Functions $f_{i}(k r)$ and, $g_{i}(k r)$ have regular and irregular behavior at the origin, respectively. For simplicity, in this paper we have assumed that the Coulomb interaction is absent, and, correspondingly, that the boundary conditions (4) include Coulomb functions with Sommerfeld parameters equal to zero. The possible inclusion of Coulomb forces in (5) is straightforward.

In the variable phase method [17-19], the solutions of Eq. (1) are written as combinations of free solutions with unknown functions $\alpha_{i n}(r)$ and $\beta_{i n}(r)$,

$$
\psi_{\text {in }}(r)=f_{i}(k r) \alpha_{i n}(r)-g_{i}(k r) \beta_{i n}(r) .
$$

The regular set of $N$ solutions can be obtained with the initial values $\alpha_{i n}(0)=\delta_{i n}$ and $\beta_{i n}(0)=0$. (We demand that at $r \rightarrow$ 0 , for the regular solution, $\psi_{n n}(r) \sim f_{n}(k r)$, while $\psi_{\text {in }}(r)$ for $i \neq n$ decrease faster than $f_{n}(k r)$. Thus, terms $g_{i}(k r) \beta_{i n}(r)$ must be negligible in comparison with $f_{i}(k r) \alpha_{i n}(r), \beta_{i n}(r) \sim$ $\left.o\left[f_{n}(k r) / g_{i}(k r)\right]\right)$. The number of unknown functions in (6) is doubled compared to the number of functions $\psi_{\text {in }}(r)$. This freedom can be eliminated by the introduction of additional constraints on $\alpha_{i n}(r)$ and $\beta_{i n}(r)$. Thus, we require that the derivatives $\psi_{\text {in }}^{\prime}(r)$ do not include derivatives of functions $\alpha_{i n}(r)$ and $\beta_{\text {in }}(r)$, and $\psi_{\text {in }}(r)$ can be written as

$$
\psi_{\text {in }}^{\prime}(r)=k\left[f_{i}^{\prime}(k r) \alpha_{i n}(r)-g_{i}^{\prime}(k r) \beta_{i n}(r)\right] .
$$

This condition is equivalent to the constraint

$$
f_{i}(k r) \alpha_{i n}^{\prime}(r)=g_{i}(k r) \beta_{i n}^{\prime}(r),
$$

and reduces (1) to a system of $2 N$ coupled ordinary differential equations of the first order for $\alpha_{i n}(r)$ and $\beta_{i n}(r)$,

$$
\begin{aligned}
& \alpha_{i n}^{\prime}(r)=\frac{1}{k} g_{i}(k r) \sum_{j=1}^{N} V_{i j}(r) \psi_{j n}(r), \\
& \beta_{i n}^{\prime}(r)=\frac{1}{k} f_{i}(k r) \sum_{j=1}^{N} V_{i j}(r) \psi_{j n}(r) .
\end{aligned}
$$

It is convenient to work with versions of the variable phase method where the ratio $\boldsymbol{M}(r)=\boldsymbol{\beta}(r) \boldsymbol{\alpha}(r)^{-1}$ of matrices $\boldsymbol{\alpha}(r)$ and $\boldsymbol{\beta}(r)$ is introduced. The ratio $\boldsymbol{M}(r)$ is invariant under linear transformation of the solution matrix $\boldsymbol{\Psi}(r)$ induced by any nonsingular constant matrix. Depending on the concrete choice of free solutions $f_{i}(k r)$ and $g_{i}(k r)$ [for example, it is possible to use their combinations $\left.h_{i}^{( \pm)}(x)=g_{i}(x) \pm \imath f_{i}(x)\right]$, these variants can be called a $K$-matrix formulation, $S$-matrix formulation, and so on. Then, $\psi_{i n}(r)$ has the following representation:

$$
\psi_{\mathrm{in}}(r)=\sum_{j=1}^{N}\left[f_{i}(k r) \delta_{i j}-g_{i}(k r) M_{i j}(r)\right] \alpha_{j n}(r),
$$

with an explicit relation between $\alpha_{j n}(r)$ and $\beta_{i n}(r)$ functions given by

$$
\beta_{i n}(r)=\sum_{j=1}^{N} M_{i j}(r) \alpha_{j n}(r)
$$


Taking the derivative of (11) using (9), a coupled system of $N$ Riccati equations for $M_{i j}(r)$ is obtained,

$$
\begin{aligned}
M_{i n}^{\prime}(r)= & \sum_{j, m=1}^{N}\left[f_{i}(k r) \delta_{i j}-M_{i j}(r) g_{j}(k r)\right] \frac{1}{k} V_{j m}(r) \\
& \times\left[f_{n}(k r) \delta_{m n}-g_{m}(k r) M_{m n}(r)\right] .
\end{aligned}
$$

This formulation requires a sequential solution of $N$ first-order equations for two systems: (12) for $M_{i n}(r)$, and then (9a) for functions $\alpha_{i n}(r)$; while formulation (9) requires solutions of the full system of $2 N$ coupled equations.

Equations (9) and (12) allow the explicit investigation of the reasons that catalyze the loss of a linear independence for different solution sets. The centrifugal barriers are an essential part of the dynamics described by radial equations. It is possible to take them into account analytically by introducing regular $f_{i}(k r)$ and irregular $g_{i}(k r)$ solutions of the free Schrödinger equations (5). Thus centrifugal barriers drop out from the consideration and their influence on the full solution is contained in the $f_{i}(k r)$ and $g_{i}(k r)$ functions of Eqs. (9) and (12). The regular and irregular functions of different orders are mixed by coupling potentials in the coupled equations. These functions have a quantitatively different radial behavior; some of them may be rather small while others are very large. The difference in absolute values can easily reach many orders of magnitude. Under such circumstances, it is difficult to keep an acceptable level of accuracy in the numerical solution of coupled equations. The lack of accuracy leads to the loss of the linear independence of different solutions. A possible way out is to make a rearrangement of coupled equations which minimizes the difference in the behavior of free solutions. There exist only three suitable combinations that can perform such a minimization: the product $f_{i}(x) g_{i}(x)$ of free functions or their logarithmic derivatives $f_{i}^{\prime}(x) / f_{i}(x)$ and $g_{i}^{\prime}(x) / g_{i}(x)$. After the rearrangement, a new system of coupled equations has significantly weaker demands for the numerical accuracy of the solutions. Such a rearrangement of Eqs. (12) and (9) is the main idea of this paper.

We introduce functions $U_{i j}(r)$ instead of $M_{i j}(r)$ by the relation

$$
U_{i j}(r)=f_{i}(k r) g_{i}(k r) \delta_{i j}-g_{j}(k r) M_{i j}(r) g_{j}(k r),
$$

transforming Eqs. (12) to the more symmetric system,

$$
g_{i}(k r) M_{i n}^{\prime}(r) g_{n}(k r)=\sum_{j, m=1}^{N} U_{i j}(r) \frac{1}{k} V_{j m}(r) U_{m n}(r) .
$$

This transformation requires that $g_{i}(k r)$ are different from zero in the region of radii where we seek solutions $\psi_{\text {in }}(r)$. By differentiating $U_{i j}(r)$ (13) and using Eq. (14), the following system of the Riccati equations for $U_{i j}(r)$ is obtained:

$$
\begin{aligned}
U_{i n}^{\prime}(r)= & k \delta_{i n}+k\left[\frac{g_{i}^{\prime}(k r)}{g_{i}(k r)}+\frac{g_{n}^{\prime}(k r)}{g_{n}(k r)}\right] U_{i n}(r) \\
& -\sum_{j, m=1}^{N} U_{i j}(r) \frac{1}{k} V_{j m}(r) U_{m n}(r) .
\end{aligned}
$$

Wave functions (10) are related to matrix $\boldsymbol{U}(r)$ by

$$
\psi_{\text {in }}(r)=\sum_{j=1}^{N} U_{i j}(r) \frac{\alpha_{j n}(r)}{g_{j}(k r)}=\sum_{j=1}^{N} U_{i j}(r) \tilde{\alpha}_{j n}(r),
$$

where the unknown functions $\tilde{\alpha}_{j n}(r)=\alpha_{j n}(r) / g_{j}(k r)$ satisfy the system of linear equations

$$
\tilde{\alpha}_{i n}^{\prime}(r)=-k \frac{g_{i}^{\prime}(k r)}{g_{i}(k r)} \tilde{\alpha}_{i n}(r)+\frac{1}{k} \sum_{j, m=1}^{N} V_{i j}(r) U_{j m}(r) \tilde{\alpha}_{m n}(r) .
$$

It now follows from (6) and (13) that regular solutions for wave functions imply the solution of Eq. (15) with initial conditions $U_{i n}(0)=0$. (The $\alpha$ is practically the unit matrix in the vicinity of zero radius. Correspondingly, the inverse matrix $\boldsymbol{\alpha}^{-1}$ is also the unit one. Therefore, the matrix $\boldsymbol{M}$ is equal to the matrix $\boldsymbol{\beta}$ and, taking into account the behavior of $\boldsymbol{\beta}$ near zero, we arrive at the initial value of the matrix $\boldsymbol{U}$ given above.) The new formulation of the variable phase method [i.e., Eqs. (15) and (17)] contains only logarithmic derivatives of free solutions, while the ordinary procedure [Eqs. (9) or (12)] mixes free solutions. As a result, coefficients of unknown functions vary over an essentially smaller scale, and the modified method has enhanced stability properties relative to the standard one.

Instead of $\tilde{\alpha}_{i n}(r)$, we can furthermore introduce functions $\hat{\alpha}_{i n}(r)=\tilde{\alpha}_{i n}(r) g_{n}(k r)$. Equations (17) are now transformed into the following system:

$$
\begin{aligned}
\hat{\alpha}_{i n}^{\prime}(r)= & k\left[\frac{g_{n}^{\prime}(k r)}{g_{n}(k r)}-\frac{g_{i}^{\prime}(k r)}{g_{i}(k r)}\right] \hat{\alpha}_{i n}(r) \\
& +\frac{1}{k} \sum_{j, m=1}^{N} V_{i j}(r) U_{j m}(r) \hat{\alpha}_{m n}(r) .
\end{aligned}
$$

The advantage of these equations compared to (17) is that only the changes in the differences of the logarithmic derivatives play a role. This also enhances the stability of the numerical solutions. From systems (15) and (18), we get functions $U_{i n}(r)$ and $\hat{\alpha}_{i n}(r)$, which allow the calculation of wave functions $\psi_{\text {in }}(r)$ from (16) and their derivatives via relation

$$
\psi_{\mathrm{in}}^{\prime}(r)=k\left[\frac{\hat{\alpha}_{i n}(r)}{g_{n}(k r)}+\frac{g_{i}^{\prime}(k r)}{g_{i}(k r)} \psi_{\mathrm{in}}(r)\right] .
$$

From (19), a relation between the logarithmic derivative matrix of the wave function $\boldsymbol{\Psi}(r)$ and the matrix $\boldsymbol{U}(r)$ can be obtained, $\boldsymbol{\Psi}^{\prime}(r) \boldsymbol{\Psi}^{-1}(r)=k\left[\boldsymbol{U}^{-1}(r)+\boldsymbol{g}^{\prime}(r) / \boldsymbol{g}(r)\right]$. Respectively, the expression for the $R$ matrix can be written as $\mathbf{R}=\boldsymbol{\Psi}(r) \boldsymbol{\Psi}^{\prime-1}(r)=(1 / k) \boldsymbol{U}(r)\left[1+\frac{\boldsymbol{g}^{\prime}(r)}{\boldsymbol{g}(r)} \boldsymbol{U}(r)\right]^{-1}$.

Applications of the modified method to bound-state and continuum problems are different in some important details and considered separately below.

\section{A. Solutions for bound states}

For negative energy $(E<0)$, solutions of the free Schrödinger equation (5) have an analytical representation via modified Bessel functions $I_{\nu}$ and $K_{\nu}$,

$$
f_{i}(x)=\sqrt{x} I_{\mathcal{L}_{i}+1 / 2}(x) \quad g_{i}(x)=\sqrt{x} K_{\mathcal{L}_{i}+1 / 2}(x) .
$$


The regular $f_{i}(x)$ and irregular $g_{i}(x)$ functions (20), respectively, increase and decrease monotonously with the increasing argument $x$. They never equal zero at finite values of $x$. Their logarithmic derivatives are well-defined functions at all radii except zero, and have similar absolute values but opposite signs. Figure 1(a) shows regular $f_{i}(x)$ and irregular $g_{i}(x)$ functions with $\mathcal{L}_{i}=3 / 2,19 / 2$, and 39/2 represented by the solid, dashed, and dash-dotted lines, respectively. (In the hyperspherical harmonics method, these values of $\mathcal{L}_{i}=$ $K_{i}+3 / 2$ correspond to calculations with the hypermoment $K_{i}=0,8$, and 18 , respectively.) We see that changes in the absolute values of free solutions can easily span 20 orders of magnitude. [Figure 1(a) shows variations from $10^{-10}$ to $10^{+10}$.] Variations of logarithmic derivatives for these functions, on the other hand, as shown in Fig. 1(b), span only a few (2-3) orders of magnitude.

For negative energies, regular solutions of Eq. (1) at large radii can have both exponentially increasing and exponentially decreasing behavior. In numerically outward integration from the origin, the exponentially increasing part will very soon dominate the solutions. Only for some discrete values of the energy can the increasing part be excluded. The practical
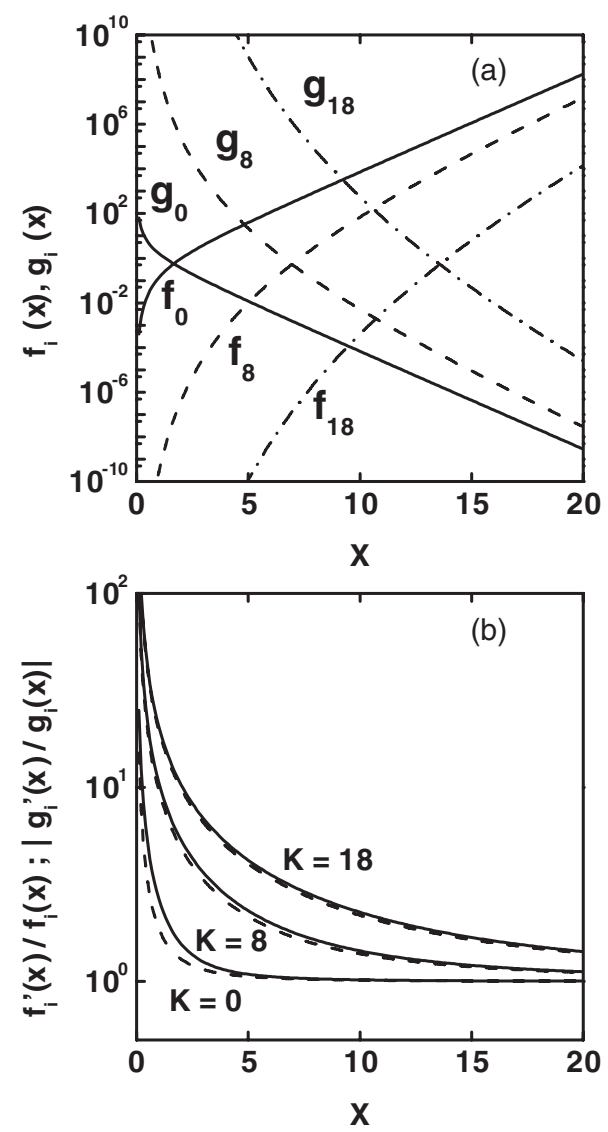

FIG. 1. (a) Regular $f_{i}(x)$ and irregular $g_{i}(x)$ solutions of the free Schrödinger equations for negative energies. The solid, dashed, and dash-dotted lines correspond to calculations with hypermoments $K=$ 0,8 , and 18, respectively. (b) Absolute values of the logarithmic derivatives of regular $f_{i}(x)$ (solid line) and irregular $g_{i}(x)$ (dashed line) solutions. way to find these energy eigenvalues is to perform the inward integration from large radii toward the origin, starting from exponentially decreasing solutions. At the origin, such solutions will have parts with regular and irregular behavior, and the irregular parts will dominate the numerical integration. We can try to match wave functions and their derivatives for inward and outward solutions at some radius. A smooth matching is possible only at discrete energy values where bound states exist. As a result, the obtained wave functions will satisfy the required boundary conditions. Within the variable phase method, a convenient way to find the inward solutions $\psi_{\text {in }}^{i n w}(r)$ is to use the following representation:

$$
\psi_{\text {in }}^{i n w}(r)=\sum_{j=1}^{N}\left[f_{i}(k r) L_{i j}(r)-g_{i}(k r) \delta_{i j}\right] \beta_{j n}(r),
$$

where the matrix $\boldsymbol{L}(r)=\boldsymbol{\alpha}(r) \boldsymbol{\beta}(r)^{-1}$ is the inverse of the matrix $\boldsymbol{M}(r)$. Then, the set of $N$ linear independent solutions with the exponentially decreasing behavior $\left[\sim g_{i}(k r)\right]$ can be obtained by the inward integration from some large radius $r_{b}$ with initial values $L_{i j}\left(r_{b}\right)=0$ and $\beta_{i n}\left(r_{b}\right)=\delta_{i n}$. The corresponding matrices $\boldsymbol{W}(r)$ and $\hat{\boldsymbol{\beta}}(r)$ can be defined as

$$
\begin{aligned}
W_{i j}(r) & =f_{j}(k r) L_{i j}(r) f_{j}(k r)-f_{i}(k r) g_{i}(k r) \delta_{i j}, \\
\hat{\beta}_{i n}(r) & =\frac{1}{f_{i}(k r)} \beta_{i n}(r) f_{n}(k r),
\end{aligned}
$$

and the following system of equations can be derived:

$$
\begin{aligned}
W_{i n}^{\prime}(r)= & k \delta_{i n}+k\left[\frac{f_{i}^{\prime}(k r)}{f_{i}(k r)}+\frac{f_{n}^{\prime}(k r)}{f_{n}(k r)}\right] W_{i n}(r) \\
& -\sum_{j, m=1}^{N} W_{i j}(r) \frac{1}{k} V_{j m}(r) W_{m n}(r), \\
\hat{\beta}_{i n}^{\prime}(r)= & k\left[\frac{f_{n}^{\prime}(k r)}{f_{n}(k r)}-\frac{f_{i}^{\prime}(k r)}{f_{i}(k r)}\right] \hat{\beta}_{i n}(r) \\
& +\frac{1}{k} \sum_{j, m=1}^{N} V_{i j}(r) W_{j m}(r) \hat{\beta}_{m n}(r) .
\end{aligned}
$$

Equations (15) and (23) [(18) and (24)] have similar structure, but only logarithmic derivatives from irregular functions $g_{i}(k r)$ appear in the first case (15) [(18)] and from regular functions $f_{i}(k r)$ in the second case (23) [(24)].

We demand that at some (matching) point $r_{m}$, a linear combination of wave functions and derivatives for both sets become equal,

$$
\begin{aligned}
& \sum_{n=1}^{N} \psi_{\text {in }}^{\text {out }}\left(r_{m}\right) \lambda_{n}=\sum_{n=1}^{N} \psi_{\text {in }}^{\text {inw }}\left(r_{m}\right) \mu_{n}, \\
& \sum_{n=1}^{N} \psi_{\text {in }}^{\text {out }^{\prime}}\left(r_{m}\right) \lambda_{n}=\sum_{n=1}^{N} \psi_{\text {in }}^{\text {inw }}\left(r_{m}\right) \mu_{n} .
\end{aligned}
$$

Here, $\left\{\lambda_{n}\right\}$ and $\left\{\mu_{n}\right\}$ are unknown mixing coefficients that must be found by solving the homogeneous system (25) of $2 \mathrm{~N}$ linear equations. A solution exists only if the determinant of the system, constructed from wave functions and derivatives, 
equals zero. This may only happen at a discrete value of the energy $E$, which is the energy of a bound state. Using the explicit representation for wave functions and their derivatives via functions $U_{i n}\left(r_{m}\right), \hat{\alpha}_{i n}\left(r_{m}\right), W_{i n}\left(r_{m}\right)$, and $\hat{\beta}_{i n}\left(r_{m}\right)$, the problem of finding energy eigenvalues is reduced to the more simple requirement that the $N \times N$ matrix $\boldsymbol{D}$,

$$
\begin{aligned}
D_{i n}= & U_{i n}\left(r_{m}\right)-W_{i n}\left(r_{m}\right) \\
& +\sum_{j=1}^{N} W_{i j}\left(r_{m}\right)\left[\frac{f_{i}^{\prime}\left(k r_{m}\right)}{f_{i}\left(k r_{m}\right)}-\frac{g_{i}^{\prime}\left(k r_{m}\right)}{g_{i}\left(k r_{m}\right)}\right] U_{j n}\left(r_{m}\right),
\end{aligned}
$$

has zero determinant, $\operatorname{det}|\boldsymbol{D}|=0$. It is important to note that the calculation of $\boldsymbol{D}$ requires only knowledge of matrices $\boldsymbol{U}$ and $\boldsymbol{W}$, which can be obtained from solutions of the $N$ first-order coupled Riccati equations. Hence we have a procedure to search for the energy of bound states: (i) First, the energy intervals, where the determinant changes sign, are defined. (ii) Then, a search for zero of the determinant within an energy interval gives the bound-state energy. Knowing sets of solutions for this energy, we can find the coefficients $\left\{\lambda_{n}\right\}$ from the system

$$
\sum_{n=1}^{N}\left[\sum_{j=1}^{N} D_{i j} \tilde{\alpha}_{j n}\left(r_{m}\right)\right] \lambda_{n}=0 .
$$

For that, we can arbitrarily fix one of the mixing coefficients $\lambda_{n}$ (for example, by putting it equal to unity) and the rest of them can be found by solving the inhomogeneous system of $(N-1)$ equations obtained from (27). The coefficients $\left\{\mu_{n}\right\}$ are defined by the matrix relation $\boldsymbol{\mu}=\boldsymbol{\Psi}^{\mathrm{irr}}\left(r_{m}\right)^{-1} \boldsymbol{\Psi}^{\mathrm{reg}}\left(r_{m}\right) \boldsymbol{\lambda}$. Finally, a bound-state wave function, obtained as a linear combination of $\psi_{\mathrm{in}}^{\text {out }}(r)$ and $\psi_{\mathrm{in}}^{\text {inw }}(r)$, is normalized.

\section{B. Solutions for continuum states}

For positive energy $(E>0)$, the solutions of the free Schrödinger equation (5) have analytical representation via Bessel functions [16] of the first $J_{v}$ and second $Y_{\nu}$ kinds,

$$
f_{i}(x)=\sqrt{\frac{\pi x}{2}} J_{\mathcal{L}_{i}+1 / 2}(x) ; \quad g_{i}(x)=-\sqrt{\frac{\pi x}{2}} Y_{\mathcal{L}_{i}+1 / 2}(x) .
$$

These functions have two radial regions with qualitatively different behavior. At small arguments, they have monotonicbehavior-like functions $f_{i}(x) g_{i}(x)$ at negative energies, but oscillate similar to cosine or sine functions at large arguments. Hence, the logarithmic derivatives of these functions will diverge at points where the functions are equal to zero, and thus they are not suitable for numerical applications. A way out of this problem is to consider another choice for irregular solutions and use the complex functions $h_{i}^{( \pm)}(x)=$ $g_{i}(x) \pm \imath f_{i}(x)$, instead of the real function $g_{i}(x)$ [note that $\left.W\left(f_{i}, h_{i}^{( \pm)}\right)=W\left(f_{i}, g_{i}\right)=-1\right]$. The real or imaginary parts of $h_{i}^{( \pm)}(x)$ can both be equal to zero, but at different arguments, hence the functions $h_{i}^{( \pm)}(x)$ always have finite but complex values, and their logarithmic derivatives are well defined. The price for this choice is that matrices $\boldsymbol{U}(r)$ and $\hat{\boldsymbol{\alpha}}(r)$ become
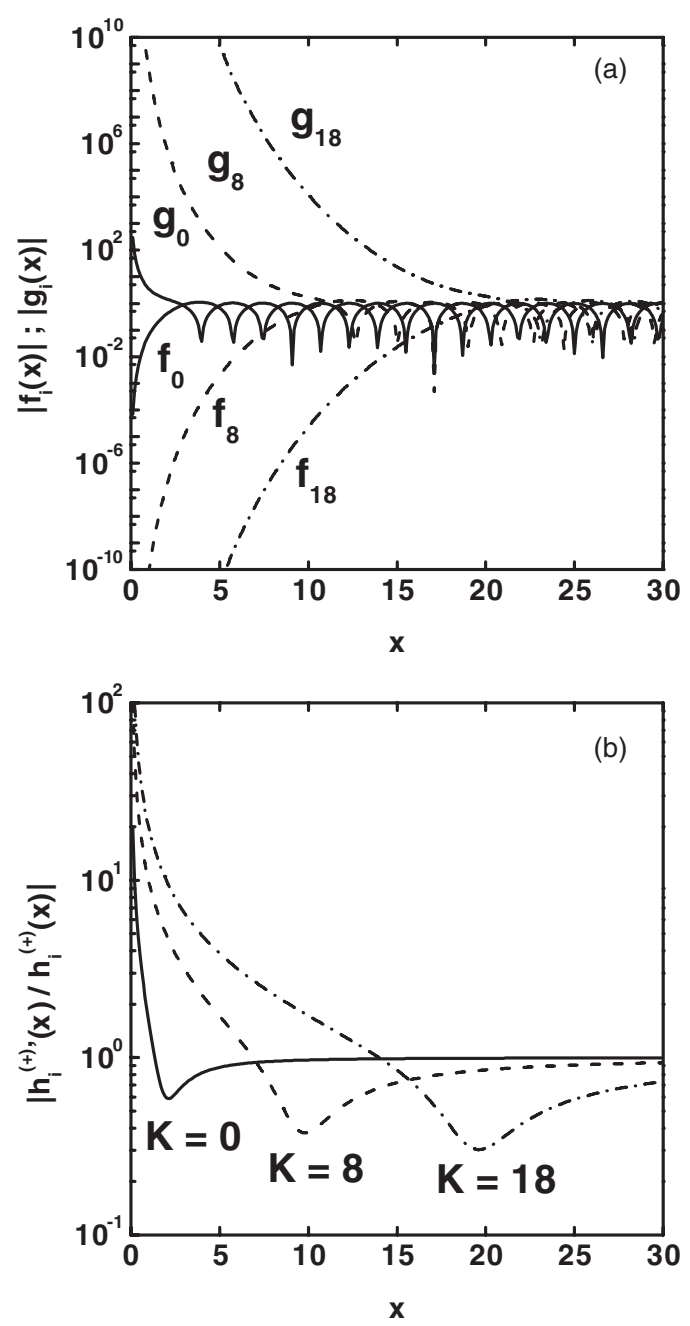

FIG. 2. Solutions of the free Schrödinger equations for positive energies: (a) regular $f_{i}(x)$ and irregular $g_{i}(x)$ functions, and (b) absolute values of the logarithmic derivatives of $h_{i}^{(+)}(x)$. The solid, dashed, and dash-dotted lines on both figures correspond to calculations with hypermoments $K_{i}=0,8$, and 18, respectively $\left(\mathcal{L}_{i}=K_{i}+3 / 2\right)$.

complex and the solutions of Eqs. (15) and (18) for complex functions require more numerical resources. The $S$-matrix and continuum states (4) are, however, always complex, hence complex solutions directly correspond to the physical nature of these states. As an example, Fig. 2(a) shows the behavior of the absolute values of regular $f_{i}(x)$ and irregular $g_{i}(x)$ solutions, while Fig. 2(b) presents modules of the logarithmic derivative for $h_{i}^{(+)}(x)$. As in the bound-state case, we observe again a drastic reduction in scale variations when logarithmic derivatives are used instead of the functions themselves.

Using the functions $h_{i}^{(+)}(x)$ as free irregular solutions, the matrix $\boldsymbol{\Psi}(r)$ of wave functions (6) can be written as

$$
\boldsymbol{\Psi}(r)=\left[\boldsymbol{f}(k r)-\boldsymbol{h}^{(+)}(k r) \boldsymbol{M}(r)\right] \boldsymbol{\alpha}(r) .
$$

Here, $\boldsymbol{f}(k r)$ and $\boldsymbol{h}^{( \pm)}(k r)$ are diagonal matrices. Respectively, the matrix $\boldsymbol{U}(r)$ from Eq. (13) is defined now as

$$
\boldsymbol{U}(r)=\boldsymbol{f}(k r) \boldsymbol{h}^{(+)}(k r)-\boldsymbol{h}^{(+)}(k r) \boldsymbol{M}(r) \boldsymbol{h}^{(+)}(k r) .
$$


Additionally, we introduce the matrix $\boldsymbol{S}(r)=\mathbf{1}-2 \imath \boldsymbol{M}(r)$, which has a simple relation with the matrix $\boldsymbol{U}(r)$,

$$
\begin{aligned}
\boldsymbol{S}(r)= & \boldsymbol{h}^{(-)}(k r)\left[\boldsymbol{h}^{(+)}(k r)\right]^{-1} \\
& +2 l\left[\boldsymbol{h}^{(+)}(k r)\right]^{-1} \boldsymbol{U}(r)\left[\boldsymbol{h}^{(+)}(k r)\right]^{-1} .
\end{aligned}
$$

Performing the integration of Eq. (15) [where $h_{i}^{(+)}(k r)$ appear instead of functions $g_{i}(k r)$ ] to the large radius $r_{b}$ where the influence of coupling potentials is negligible, the matrix $S(r)$ becomes a constant matrix $S$ and its elements $S_{i n}$ define the asymptotic behavior (4) of the scattering wave functions. In the case of real potentials, the $\boldsymbol{S}$ matrix has to be unitary, $S \boldsymbol{S}^{\dagger}=\mathbf{1}$. This property puts rather strict constraints not only on the symmetry of respective matrix elements, but also on their absolute values. The unitarity of the $S$ matrix is not explicit in the solutions of Eq. (15), and the fulfillment of this property is a good check of the calculation accuracy. Moreover, the matrix $\boldsymbol{S}(r)$ must be unitary at all radii for real potentials. Practice shows that solutions of the system (15) are rather stable with an increasing number of equations and the propagation to large radii.

The continuum states $\boldsymbol{\Psi}\left(r_{b}\right)$ at large radius $r_{b}$ can be normalized by a constant matrix $\lambda$ to the asymptotic shape (4),

$$
\begin{aligned}
\boldsymbol{\Psi}\left(r_{b}\right) \lambda & =\boldsymbol{f}\left(k r_{b}\right)-\boldsymbol{h}^{(+)}\left(k r_{b}\right)(\mathbf{1}-\boldsymbol{S}) / 2 l, \\
\boldsymbol{\Psi}^{\prime}\left(r_{b}\right) \lambda & =k\left[\boldsymbol{f}^{\prime}\left(k r_{b}\right)-\boldsymbol{h}^{(+)^{\prime}}\left(k r_{b}\right)(\mathbf{1}-\boldsymbol{S}) / 2 l\right] .
\end{aligned}
$$

Using relations (16) and (19), we can obtain from Eqs. (32) the normalization matrix $\boldsymbol{\lambda}=\boldsymbol{h}^{(+)}\left(k r_{b}\right) \hat{\boldsymbol{\alpha}}^{-1}\left(r_{b}\right)\left[\boldsymbol{h}^{(+)}\left(k r_{b}\right)\right]^{-1}$ and connection (31) between the $\boldsymbol{S}$ and $\boldsymbol{U}$ matrices.

If we need not only the $S$ matrix but also scattering wave functions, then the matrix $\hat{\alpha}(r)$ from Eq. (18) has to be calculated in addition to $\boldsymbol{U}(r)$. Practice shows that the calculations of $\hat{\boldsymbol{\alpha}}(r)$ are more troublesome compared to $\boldsymbol{U}(r)$ calculations. If the number and the value of orbital angular momenta $\mathcal{L}_{i}$ included in the system are increased, then a loss of the linear independence in outward integration may appear at some radius. This is a consequence of two factors: (i) the radial region, where the integration is under barriers, becomes large, and (ii) the difference in absolute values of various components increases. This can be formulated more precisely: The region $\left(r<\mathcal{L}_{i} / k\right)$, where functions $f_{i}(k r)$ and $g_{i}(k r)$ have monotonic and very diverse behavior, increases with increasing $\mathcal{L}_{i}$ values and can extend over tens of femtometers. At conditions when coupling potentials are slowly decaying (this is the case for halo nuclei), a numerical stability in the process of long radial integration needed for reaching the wave function asymptotic may gradually deteriorate in spite of the enhanced stability of the modified equations. With increasing $\mathcal{L}_{i}$, the loss of accuracy develops faster and, respectively, the radius where an instability may appear becomes smaller. There is a practical way to detect instabilities: If linear independence is lost at some radius, then the inverse matrix of $\hat{\boldsymbol{\alpha}}(r)$ is poorly defined. Thus, checking the identity $\hat{\boldsymbol{\alpha}}(r) \hat{\boldsymbol{\alpha}}^{-1}(r)=\mathbf{1}$ allows one to establish a radius $r_{o}$ up to which the outward calculation of $\hat{\boldsymbol{\alpha}}(r)$ is still reliable.

If numerical instabilities of the matrix $\hat{\boldsymbol{\alpha}}(r)$ were detected in the process of the outward integration, then wave function calculations for radii larger then $r_{o}$ can be made by another procedure. The matrix $\boldsymbol{U}(r)$ is known for all radii from the origin up to the maximal radius $r_{b}$. Therefore, from the matrix $\boldsymbol{U}\left(r_{b}\right)$, we know the $\boldsymbol{S}$ matrix and the wave function asymptotic (4), which can be written as $\boldsymbol{\Psi}_{\text {inw }}\left(r_{b}\right)=\boldsymbol{U}\left(r_{b}\right)\left[\boldsymbol{h}^{(+)}\left(k r_{b}\right)\right]^{-1}$, respectively. Demanding that wave functions (16) equal the asymptotic (4), we obtain initial values of $\hat{\alpha}_{i n}\left(r_{b}\right)=\delta_{i n}$, and can perform inward integrations of Eq. (18) with the already calculated matrix $\boldsymbol{U}(r)$ from $r_{b}$ to $r_{o}$. Instabilities may also appear in the inward integration, but they develop much more slowly since the most dangerous region is at small radii. Again, the linear independence of solutions can be checked at $r_{o}$. Finally, wave function $\Psi_{\text {out }}(r)$ obtained in outward integration from the origin up to $r_{o}$ is normalized to inward integrated wave function $\boldsymbol{\Psi}_{i n w}\left(r_{o}\right)$, which has a correct normalization, $\boldsymbol{\Psi}_{\text {out }}\left(r_{o}\right) \boldsymbol{\eta}=\boldsymbol{\Psi}_{\text {inw }}\left(r_{o}\right)$. From this matrix relation, we get the mixing coefficients $\boldsymbol{\eta}$, $\boldsymbol{\eta}=\boldsymbol{h}^{(+)}\left(k r_{o}\right) \hat{\boldsymbol{\alpha}}_{\text {out }}^{-1}\left(r_{o}\right) \hat{\boldsymbol{\alpha}}_{\text {inw }}\left(r_{o}\right)\left[\boldsymbol{h}^{(+)}\left(k r_{o}\right)\right]^{-1}$. In this way, the correctly normalized wave function for all necessary radii can be calculated.

\section{DISCUSSION}

The centrifugal potentials are an important part of the dynamics described by the system of coupled radial Schrödinger equations. The numerical solutions in the regions where motions are under barriers lead to the mixing of large and small components that coexist at these conditions. When the accuracy of numerical integration is not enough for tracing different solutions, such mixing may lead to the loss of the linear independence. This is one of the major problems in the numerical solutions of the coupled system of equations. In the variable phase method, the regular and irregular solutions of the free Schrödinger equation take centrifugal barriers implicitly into account. As a result, centrifugal barriers drop out from the final system of the first-order differential equations, and their influence appears only via free solutions that can, however, differ in the magnitude by many orders. Thus, the system of coupled equations contains terms that are very different on absolute values, and numerical instabilities may develop when the solution accuracy falls short. The modification of the variable phase method suggested here tries to remedy this. The modification consists of a rearrangement of equations into a set which includes only the logarithmic derivatives of free solutions. Since the variations of the magnitude of the logarithmic derivatives are essentially smaller compared to the absolute-value variation of free solutions, the conditions for developing numerical instabilities are strongly suppressed.

Below we demonstrate the modified method by solving a concrete physical problem, namely, by calculating wave functions for light halo nuclei within a cluster few-body model [20]. The method of the hyperspherical harmonics is very convenient for the description of the three-body structure of two-neutron halos that appear in Borromean nuclei ${ }^{6} \mathrm{He}$, ${ }^{11} \mathrm{Li}$, etc., at the very edge of the nuclear stability (see recent works [21,22], and references therein, for a more detailed discussion of the successes and challenges of this approach). The relative motion of three clusters is described in the space of 


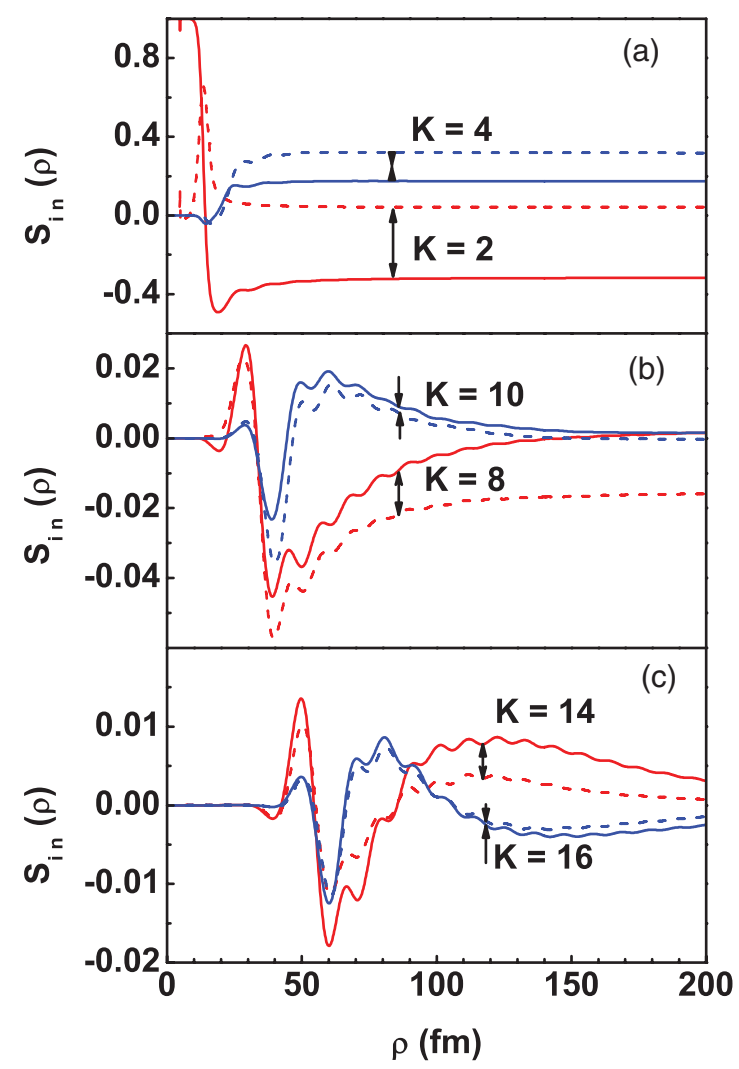

FIG. 3. (Color online) The real (solid line) and imaginary (dashed line) parts of the $S(\rho)$-matrix elements as a function of the hyperradius $\rho$ for the $J^{\pi}=0^{+}$continuum state of the ${ }^{6} \mathrm{He}$ nucleus at excitation energy $E=2 \mathrm{MeV}$. All matrix elements have the same quantum numbers $\left(S=L=l_{x}=l_{y}=0\right.$ ) but different hypermoment $K$. The ingoing channel has $K=2$, while outgoing channels have $K=$ (a) 2 and 4 , (b) 8 and 10, and (c) 14 and 16. Note the different vertical scales for the (a), (b), and (c) cases.

hyperspherical coordinates $\left(\rho, \Omega_{5}\right)$, and the nuclear wave function $\Psi^{J \pi}$ is decomposed on a basis of hyperspherical harmon$\operatorname{ics} \Upsilon_{K v}\left(\Omega_{5}\right)[20], \Psi^{J \pi}=\sum_{K v} \psi_{K v}^{J \pi}(\rho) \Upsilon_{K v}\left(\Omega_{5}\right)$. Here, $\rho, \Omega_{5}$, and $K$ are the hyperradius, hyperangles, and hypermoment, respectively. The index $v$ denotes all quantum numbers in addition to $K$, which are needed for a complete identification of the basis. If the Schrödinger equation for the wave function $\Psi^{J \pi}$ is multiplied by hyperspherical harmonics $\Upsilon_{K v}\left(\Omega_{5}\right)$ from the left and integrated over hyperangles $\Omega_{5}$, a system of coupled hyperradial differential equations similar to (1) is obtained. In this system, $\mathcal{L}_{i}=K_{i}+3 / 2$ are the generalized orbital angular momenta, where $K_{i}$ is the hypermoment in the $i$ th channel, and the matrix elements $V_{i j}(\rho)$ (containing all intercluster interactions) in the basis of hyperspherical harmonics depend only on the hyperradius $\rho$. More details about the development of the model and applied interactions can be found in [23].

As an example, we consider the calculation of the $0^{+}$ continuum wave function of the ${ }^{6} \mathrm{He}$ nucleus at excitation energy $2 \mathrm{MeV}$. All possible hyperharmonics up to the value of $K=20$ are included in the wave function expansion, giving a system of Schrödinger equations with $\sim 70$ coupled channels.
Figure 3 shows several elements of the $\boldsymbol{S}(\rho)$ matrix given in (31) as a function of the hyperradius $\rho$. All elements have the same angular momentum quantum numbers $L=S=$ $l_{x}=l_{y}=0$, but different values of hypermoment $K$, and correspond to the ingoing channel with $K=2$. In this case, the ingoing channel corresponds to a low centrifugal barrier and the flux due to the coupling is distributed between different channels. Channels with larger $K$ values are centrifugally more strongly suppressed at small radii, as clearly seen in Figs. 3(b) and 3(c). When the potentials become negligible with increasing hyperradius $\rho$, the $\boldsymbol{S}(\rho)$-matrix elements are seen to take constant asymptotic values. In the method of hyperspherical harmonics, coupling potentials decay slowly with the hyperradius (in our case, $\sim 1 / \rho^{3}$ ), and to reach the asymptotic region, the integration has to be continued to rather large radii. For example, components with $K \leqslant 4$ are stabilized at about $50 \mathrm{fm}$, while those with $K \sim 10$ need $\rho \sim 150 \mathrm{fm}$, and components with larger $K$ values can require even larger distances.

Figure 4 shows the same $\boldsymbol{S}(\rho)$-matrix elements, but for the case when the ingoing flux is in a channel with a large centrifugal barrier $(K=16)$. At small radii, the barrier dominates and the ingoing wave cannot penetrate to small distances where coupling potentials are large. As a result, the flux is kept mainly in the ingoing channel. Only at large radii $(\sim 50 \mathrm{fm})$ does the flux start to redistribute between different channels. But at large radii, the coupling potentials become

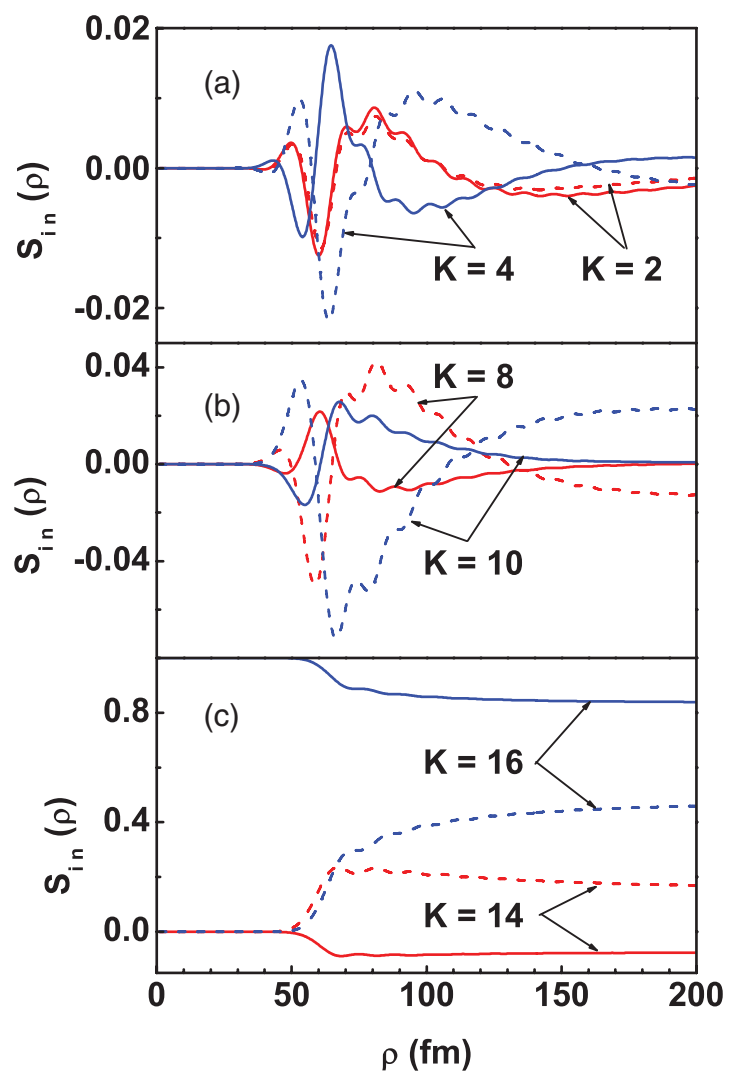

FIG. 4. (Color online) $S(\rho)$-matrix elements as a function of hyperradius $\rho$ with ingoing channel at $K=16$. Notations are the same as in Fig. 3. 
relatively weak and cannot significantly populate channels different from the incoming one.

This example serves as an illustration of the suggested method. Some questions, important for practical applications, are not discussed in this paper; for example, which particular numerical methods are more suited for solving the system of equations, and so on. Different answers exist and practical prescriptions should be optimized for any concrete model. To cover these issues, the physical models have to be explicitly formulated. Since our main aim was to present the general idea of the method, these important practical questions will have to be illuminated elsewhere.

\section{CONCLUSION}

In numerical solutions, the Schrödinger equation is often converted to a coupled system of radial equations. The internal dynamics of such a system may be very versatile and complicated due to coupling potentials, but also carries general features due to the universality of the kinetic energy operator. These universal properties are contained in different centrifugal barriers and lead to the appearance of difficulties in the numerical solutions of coupled equations in radial regions where the motion for some channels is classically forbidden. Such classically forbidden regions exist even in cases when coupling potentials are absent, and solutions within such regions are described by Bessel functions with known analytical properties. The absolute scales for free solutions may be very different. Coupling potentials mix and modify free solutions with widely different absolute values when they are propagated via forbidden regions. If numerical accuracy is not high enough, the propagation leads to the development of numerical instabilities in the solution vectors, and the loss of the linear independence for different sets of solution vectors.

The modified variable phase method suggested in this paper rearranges the coupled equations to a form which contains free solutions only as logarithmic derivatives, i.e., a combination which minimizes variations of absolute values. As a result, the new system is less prone to develop numerical instabilities. The method is powerful for both bound and continuum states. We have demonstrated its usefulness by computing a low-lying monopole continuum state of the Borromean halo nucleus ${ }^{6} \mathrm{He}$ in a three-body cluster model using a hyperspherical harmonic expansion.

\section{ACKNOWLEDGMENTS}

S.N.E. acknowledges financial support from the University of Bergen.
[1] R. G. Gordon, J. Chem. Phys. 51, 14 (1969).

[2] R. A. White and E. F. Hayes, J. Chem. Phys. 57, 2985 (1972).

[3] L. Gr. Ixaru, Comput. Phys. Commun. 20, 97 (1980).

[4] T. N. Rescigno and A. E. Orel, Phys. Rev. A 25, 2402 (1982).

[5] L. D. Tolsma and G. W. Veltkamp, Comput. Phys. Commun. 40, 233 (1986).

[6] B. R. Johnson, J. Chem. Phys. 69, 4678 (1978).

[7] J. M. Hutson, Comput. Phys. Commun. 84, 1 (1994).

[8] L. S. Ferreira, E. Maglione, and R. J. Liotta, Phys. Rev. Lett. 78, 1640 (1997).

[9] P. G. Burke, A. Hibbert, and W. D. Robb, J. Phys. B 4, 153 (1971).

[10] J. C. Light and R. B. Walker, J. Chem. Phys. 65, 4272 (1976).

[11] J. Tennyson, Phys. Rep. 491, 29 (2010).

[12] I. J. Thompson, F. M. Nunes, and B. V. Danilin, Comput. Phys. Commun. 161, 87 (2004).

[13] A. Deloff, Ann. Phys. 322, 1373 (2007).

[14] Z. A. Anastassi and T. E. Simos, Phys. Rep. 284, 1 (2009).
[15] S. N. Ershov, J. S. Vaagen, and M. V. Zhukov, Phys. At. Nucl. 74, 1151 (2011).

[16] Handbook of Mathematical Functions, edited by M. Abramowitz and I. A. Stegun (Dover, New York, 1964).

[17] F. Calogero, Variable Phase Approach to Potential Scattering (Academic, New York, 1967).

[18] V. V. Babikov, Method of Phase Functions in Quantum Mechanics, 2nd rev. (Nauka, Moscow, 1976).

[19] R. Martinazzo, E. Bodo, and F. A. Gianturco, Comput. Phys. Commun. 151, 187 (2003).

[20] M. V. Zhukov et al., Phys. Rep. 231, 151 (1993).

[21] B. V. Danilin, N. B. Shul'gina, S. N. Ershov, and J. S. Vaagen, Phys. At. Nucl. 72, 1324 (2009).

[22] S. N. Ershov, L. V. Grigorenko, J. S. Vaagen, and M. V. Zhukov, J. Phys. G 37, 064026 (2010).

[23] B. V. Danilin, I. J. Thompson, M. V. Zhukov, and J. S. Vaagen, Nucl. Phys. A 632, 383 (1998). 